\title{
Utilizing slope method as an alternative data analysis for functional near-infrared spectroscopy-derived cerebral hemodynamic responses
}

\author{
Kevin Mandrick $^{\mathrm{a}, \mathrm{b}}$, Gérard Derosiere ${ }^{\mathrm{a}}$, Gérard Dray ${ }^{\mathrm{c}}$, Denis Coulon ${ }^{\mathrm{b}}$, \\ Jean-Paul Micallef ${ }^{\mathrm{a}, \mathrm{d}}$, Stéphane Perrey ${ }^{\mathrm{a}, *}$ \\ ${ }^{a}$ Movement to Health (M2H), Montpellier-1 University, EuroMov, 700 Avenue du Pic Saint Loup, 34090 Montpellier, France \\ ${ }^{\mathrm{b}}$ Bodysens, 442 Rue Georges Besse, Immeuble Innovation 3, 30035 Nîmes, France \\ ${ }^{c}$ LGI2P, Ecole des Mines d'Alès site EERIE, Parc Scientifique Georges Besse, 69 Rue Georges Besse, F30035 Nîmes Cedex 1, France \\ d INSERM, ADR 08, 60 Rue de Navacelles, 34394 Montpellier Cedex 5, France
}

\begin{abstract}
A B S T R A C T
The purpose was to propose an alternative data analysis for functional near-infrared spectroscopy (fNIRS)-derived hemodynamics as a function of cortical activation changes. We evaluated hemodynamic responses from the prefrontal cortex region while 38 participants performed a cognitive task. The task consisted of an arithmetic calculation with three levels of complexity (i.e., easy, medium and difficult). These task-dependent hemodynamic responses were analyzed by the slope method (i.e. using a linear regression through the cognitive task) and were compared with the corresponding responses obtained with a traditional approach of the amplitude method. Subjective scales of task loading (assessed by DP15 and NASA-TLX) and behavioral outcomes (performance and reaction time) were also recorded in response to the task complexity. Results revealed that the proposed slope method allowed a better discrimination in terms of cortical activation among all levels of mental workload. There was no significant increase in cortical activation between the medium and difficult levels ( $p=.30 ; d=0.09)$ with the amplitude method while the slope coefficient was sensitive to the different levels $(p<.01 ; d=0.32)$. These preliminary results from a large sample size demonstrated that the slope method appears suitable for discriminating the changes in cortical activation with respect to the mental workload.

Relevance to industry: In this work, we proposed an optimum way of quantifying the mental workload of participants in terms of fNIRS-derived cerebral hemodynamic responses.
\end{abstract}

Keywords:

Near-infrared spectroscopy

Prefrontal cortex

Cognitive task

Slope

Quantitative measure

Mental workload

\section{Introduction}

A wide variety of daily tasks at work influence operators' mental load to meet task demands (Ryu and Myung, 2005; Ayaz et al., 2012). For instance, aircraft pilots need to allocate cognitive resources during landing approach and actual landing tasks (Takeuchi, 2000). Human behavior in complex task involves high brain resources, especially from the prefrontal cortex (PFC) area (Perrey et al., 2010). PFC is particularly well-described as being involved in the sophisticated neural processing and executive cognitive operations in humans (Gruber et al., 2001). Terminology

Abbreviations: ANOVA, analyses of variance; DP15, perceived difficulty of the task; EEG, electroencephalography; fNIRS, functional near-infrared spectroscopy; NASA-TLX, nasa task load index; PFC, prefrontal cortex; RT, reaction time; STAI, State-trait anxiety inventory.

* Corresponding author. Tel.: +33 411759 066; fax: +33 411759050

E-mail address: stephane.perrey@univ-montp1.fr (S. Perrey). in mental workload research has its roots in cognitive and physiological theories. Workload is the result of reaction to demand; it is the proportion of the capacity that is allocated for task performance. This construct is generally interpreted by both temporal demand and cognitive costs over the brain state (Pickup et al., 2010). The first is based on measures of reaction time (RT) and the second is based on measures of performance accuracy when performing a task. In other part, multidimensional subjective measurement techniques are valuable to assess mental workload (e.g., NASA-TLX). Finally, the mental load can be directly quantified by various psychophysiological measures (e.g., cardiac and respiratory activity, ocular blinking, skin sweating, etc.) or by the level of brain activity required for performing a given task (Fairclough et al., 2005; Ryu and Myung, 2005; Perrey et al., 2010). Hence, the level of brain activation might be related to a proportional mental workload associated to the level of the task difficulty.

Currently, the main neuroimaging techniques measure electrophysiological or hemodynamic signals over the scalp (e.g., 
electroencephalography, (EEG) or functional near-infrared spectroscopy, (fNIRS), respectively). Di Nocera et al. (2007) stipulated "it is well known that some EEG bandwidths and components of the event-related potentials are sensitive to variations in mental workload. However, these indices are difficult to collect and analyze in real time, and their reliability and stability are seldom assessed. Moreover, real-time filtering to obtain single-trial data is still an issue that is far from being solved." Recently, some studies showed that the mental workload-related PFC activity can be alternatively assessed with fNIRS (Ayaz et al., 2012; Hirshfield et al., 2009; Izzetoglu et al., 2004, 2007; Perrey et al., 2010). fNIRS is a wellestablished noninvasive optical technique for estimating cortical activation and has the advantage to be weakly insensitive to motion artifact (Perrey, 2008). Thus, fNIRS appears suitable for detecting hemodynamic changes in real time while the subject is performing a functional task (e.g., sensory stimulation, cognitive task or physical exercise, León-Carrión and León-Domínguez, 2012; Perrey et al., 2010). A cortical activation from fNIRS signals is usually characterized by a large increase in oxyhemoglobin (oxy-Hb) with a lower delayed drop in deoxyhemoglobin (deoxy-Hb) (Ferrari and Quaresima, 2012; Gervain et al., 2011; Perrey, 2008, Perrey et al., 2010). In reality, the activation pattern of the fNIRS response turns out to be more variable and complex (Sato et al., 2005).

For example, Bauernfeind et al. (2008) observed inverse fNIRS response characterized by an increase of deoxy-Hb and a decrease of oxy-Hb over the left PFC with different mental arithmetic tasks (one subtraction and repetitive subtractions). Yang et al. (2009) did not find any difference between the fNIRS response of the bilateral PFC as function of two subtraction tasks with distinct difficulty levels. Using low, medium and high level of mental calculation tasks, Tsunashima et al. (2012) noticed that fNIRS changes over the bilateral PFC became larger as the task difficulty increased. These discrepancies may be explained by the differences in fNIRS signal processing. Prior to demonstrating such statistical comparisons between the tasks, Tsunashima et al. (2012) post-transformed the fNIRS signals (decomposition and reconstruction) using the discrete wavelet-based multi-resolution process. Then, in order to be used statistically they proposed the Z-scored signal for converting fNIRS data into a level of workload. For details about the effectiveness of this approach and analysis see Tsunashima et al. (2012). One drawback of this study was that the authors did not compare their processing method with a more traditional fNIRS analysis to judge its sensitivity.

Usually, the magnitude of the cortical activation level relies on the significance of data changes (i.e., pre-stimulation vs. stimulation) (Gervain et al., 2011). Commonly, the main variables of interest calculated to depict the fNIRS activation pattern are: difference of oxy-Hb and deoxy-Hb signal changes (Izzetoglu et al., 2004); sum of oxy-Hb and deoxy-Hb signal changes (Izzetoglu et al., 2004; Gervain et al., 2011); area under the curve (Limongi et al., 2009; Gagnon et al., 2012); Z-score (Tsunashima et al., 2012); amplitude response for oxy-Hb and deoxy-Hb (Perrey, 2008; Holper et al., 2009; Gervain et al., 2011) by comparing a baseline period to (i) the largest response obtained during a suitable temporal window during the stimulation period (Colier et al., 1999; Gervain et al., 2011) or (ii) the mean activation values measured throughout task (Tanida et al., 2004, 2007, 2008). However, the field of fNIRS still suffers from the lack of a commonly accepted standard by which investigators could describe and compare activation magnitudes. In fact, there is no real consensus in the literature on how to analyze the fNIRS data and construe for appropriate statistics. The sensitivity of the fNIRS experiment, judged on the basis of its ability to detect subtle activations is a function of not only the magnitude of the signal change, but also the dynamical fluctuation in the background signal. For the purpose of cognitive state assessment, the rate of change in blood oxygenation of fNIRS signals was shown to be sensitive to task load changes (Izzetoglu et al., 2004; Perrey et al., 2010; Tsunashima et al., 2012). Accordingly, a linear regression slope could be more powerful and simple for assessing the rate of change in the fNIRS signals in response to cognitive tasks. The quantification of the linear regression slope over the time course of the stimulation period offers advantages in computational speed and adaptability to real-time applications. This can be an alternative method for discriminating the typical brain oxygenation responses. Normally, the cortical activation pattern is expected to depict a positive slope for oxy-Hb accompanied by a lesser negative slope for deoxy-Hb.

The purpose of this study was to propose an alternative data analysis for fNIRS-derived hemodynamics as a function of cortical activation changes. We hypothesized that the slope method as compared to a more traditional method (amplitude response) is suitable and sensitive enough for discriminating the changes in cortical activation with respect to the mental workload during cognitive tasks.

\section{Materials and methods}

\subsection{Participants}

Thirty-eight healthy, female $(n=13)$ and male $(n=25)$ volunteer adults took part in the present study (age $29.8 \pm 8.7$ years; height $1.73 \pm 0.1 \mathrm{~m}$; weight $68.1 \pm 12.6 \mathrm{~kg}$ ). We recruited people with different levels of academic achievement and various professional occupations. The participant's history and physical examination were negative for known injuries, neurological, endocrinological, respiratory and cardiovascular diseases or medication, which might affect brain function or perfusion. Each subject provided written informed consent prior to enrolling in this study. All procedures were approved by the local Ethics Committee (CPP ANSM Sud Méditérannée III, number 2010-11-05, Montpellier, France) conforming to the Declaration of Helsinki for human experimentation.

\subsection{Experimental design and procedures}

The experiment was conducted in a quiet room with no natural lighting (i.e., no window). A fluorescent tube provided the only illumination. The subjects were first seated in a very comfortable lounge chair with a headrest. This setup was followed by an explanation of the protocol before equipping them with optical NIRS sensors. Then, a session of familiarization for cognitive stimulation was proposed to the subjects with their eyes closed while staying as focused as possible. We employed a classical arithmetic task to stimulate cortical activation (Limongi et al., 2009; Sakatani et al., 2010; Tanida et al., 2004, 2007, 2008). The participants were asked to perform mathematical subtraction mentally as quickly as possible in within $60 \mathrm{~s}$. For the testing session, the randomized experimental design was divided into three main levels of task difficulty (i.e., easy, medium and difficult) with three trials for each level. The timelines and running order of the testing session (blocks design) are shown in Fig. 1. We decided to use this type of experimental design to avoid ordering effects. The easy level consisted of subtracting numbers (comprised from 1 to 9 ) to a four-digit number sequentially (e.g., $3787-7=?-4=?-9=$ etc.). Following each answer given by the subject, the investigator repeated the new four-digit number. The medium level consisted of subtracting serially a two-digit number (comprised only from 10 to 20) from a four-digit number (e.g., 3787$17=?-14=?-19=$ etc.) (Limongi et al., 2009; Sakatani et al., 2010; Tanida et al., 2004, 2007, 2008). For the difficult level, the calculation consisted of subtracting a number between 20 and 100 (e.g., 3787- 


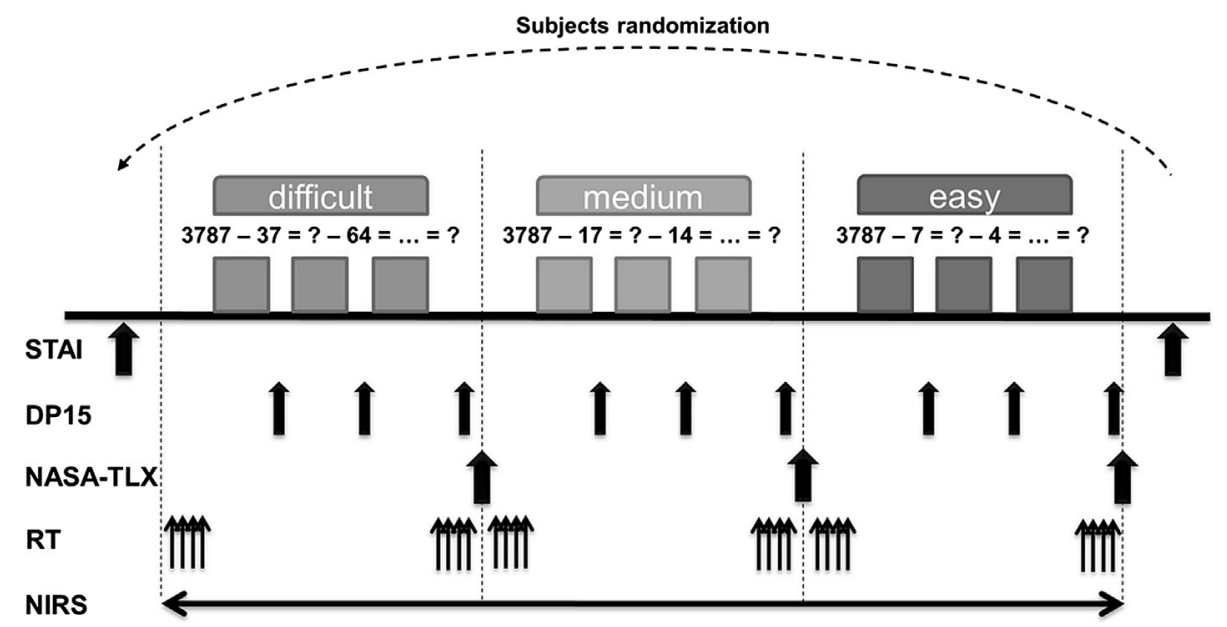

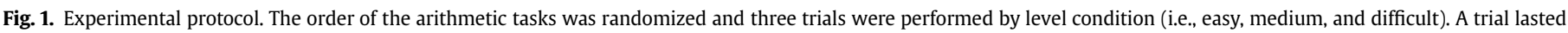

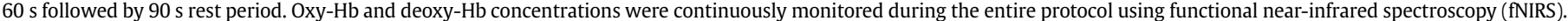

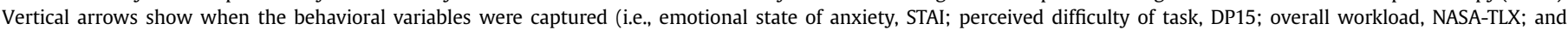
reaction time, $\mathrm{RT}$ ).

$37=?-64=?-29=$ etc.). The task performance for each level was evaluated by (i) total number of responses, (ii) number of correct answers, and (iii) percent of error rate. Prior to and immediately at the end of each trial, a reaction time (RT) task performance was measured. We asked the subjects to push a button as fast as possible after having heard an auditory stimulus delivered through a headphone. Four short "click sounds" were proposed in a random manner with a randomized 10-30 s interval inter-stimulus. The RT task permitted us to evaluate the level of focused sustained attention of the subjects after each level of difficulty of the calculation task. Moreover, after each trial, subjects were requested to assess their perceived difficulty of the arithmetic task using the DP15 scale (Delignieres et al., 1994). The DP15 scale consists of a 15-points category scale, from "extremely easy" to "extremely difficult", symmetrically placed around a central label "somewhat difficult". Also, the subjects rated their subjective workload using a French translation version of the NASA Task Load Index (NASA-TLX) at three intervals throughout the study (Hart and Staveland, 1988). The NASATLX self-reported assessment tool permitted us to evaluate the overall workload associated with the difficulty of the different mathematical calculations. This index follows a multi-dimensional rating procedure and provides a workload value based on a weighted average of ratings on six subscales (i.e., mental, physical and temporal demand, performance, effort and frustration). Finally, the anxiety state was estimated at the beginning and ending of the protocol using the state form of Spielberger's State-Trait Anxiety Inventory (STAI, form Y-A), a 20-item self-administered questionnaire (Spielberger et al., 1970).

\section{3. fNIRS data collection and processing}

In the present study, we used a commercial spectrophotometer (NIRO-200, Hamamatsu Photonics K.K., Japan) to measure the time course of relative changes in the concentrations of oxy-Hb and deoxy-Hb. The sampling rate was set at $6 \mathrm{~Hz}$. A pair of optodes was bilaterally placed over the two PFCs (dorsolateral and frontopolar areas). The optodes midpoint was placed at $3 \mathrm{~cm}$ above the centers of the upper edges of the bilateral orbital sockets, similar to 10 20 EEG system midpoint between locations Fp1/F3 and Fp2/F4, as suggested by Tanida et al. (2007). To reduce any artifact, subjects were asked to minimize head and body movements, and were given instructions to breathe gently and regularly. The NIRS data output was marked at the start of each task segment to ensure that data corresponded to the relevant epoch of the task.

Filtering, pre-processing and analysis for NIRS were performed off-line using a customized code implemented in Matlab 7.0 software (The Mathworks Inc, MA, USA). The raw NIRS data were first filtered using a first-order Butterworth low-pass filter zero-lag (cut-off frequency of $0.7 \mathrm{~Hz}$ ), thereby removing excessive high frequencies that were not due to normal physiology activity. No detrend function and offset readjustment were performed on the resulting fNIRS signals. With respect to the goal of this study, two processing methods for NIRS signals were used. For both methods, three trials were averaged for each level of task complexity (i.e., easy, medium and difficult). Fig. 2 shows a comparison of fNIRS signals with the two proposed methods.

First, changes in oxy- $\mathrm{Hb}$ and deoxy-Hb during the cognitive tasks were calculated by the difference between the rest condition (mean value of the last $10 \mathrm{~s}$ of each 1-min baseline) and the last part of the each artifact free stimulation period (mean value over the last $10 \mathrm{~s}$ ). We elected a 10-s window based on the response pattern of the NIRS signals (typical example proposed in Fig. 2 with a plateau occurrence) and studies focusing on cortex oxygenation responses to cognitive or motor tasks (Colier et al., 1999; Holper et al., 2009; Gervain et al., 2011). In case of a long stimulus period ( $\geq 1 \mathrm{~min}$ ), the oxy-Hb signal reaches a plateau that is maintained until the cessation of the stimulus (Heekeren et al., 1997).

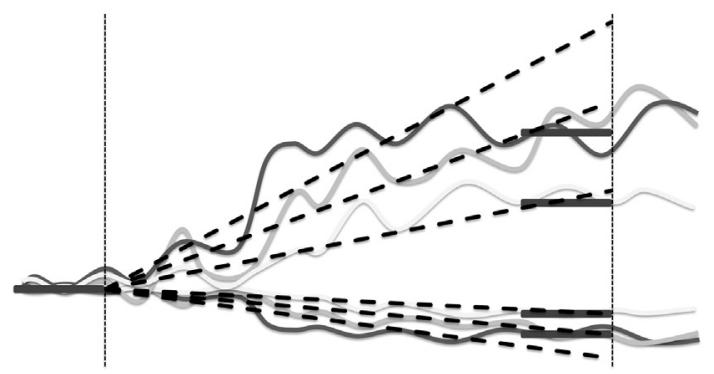

Fig. 2. Typical subject example of filtered fNIRS oxy-Hb and deoxy-Hb signals in response to the easy (in white), medium (in light gray) and difficult (in dark gray) arithmetic tasks. The period of stimulation lasted $60 \mathrm{~s}$. Dark dotted lines show the linear slope over the time course of the stimulation (method 1) whereas horizontal bars show the amplitude of changes related to $10 \mathrm{~s}$ window-average to pre-stimulation baseline of $10 \mathrm{~s}$ (method 2). 
Second, as an alternative method, the rate and amplitude of changes in oxy-Hb and deoxy-Hb during the time course of the task (i.e., over the stimulation period) were calculated by fitting the data with a linear regression technique in order to obtain the slope coefficient (see Fig. 2). The slope coefficient was indicative of the magnitude (and the direction) of the oxygenation responses over the stimulation period for each task. For example, a higher (and positive) slope value for oxy-Hb indicates a larger and faster cortical activation.

\subsection{Statistics}

All statistical analyses were performed with Statistica software (StatSoft France, 2006; version 7.1, France). Data were examined for normality and homogeneity using Shapiro-Wilk and Levene tests, respectively. Pre and post comparisons of STAI-YA scores were carried out with Student's paired $t$-test. Concerning the reaction time and the arithmetic task performance, Friedman univariate analyses of variance (ANOVA) were performed. For all other variables, no substantial deviations were observed thus permitting the use of parametric statistical analyses. DP15, NASA-TLX scores by difficulty levels were analysed with ANOVA. Two-way repeated measures ANOVA was applied to oxy-Hb and deoxy-Hb concentration as a dependent variable, with factors of hemisphere (right and left PFC) and the task level (easy, medium and difficult). When there was a significant main effect or interaction, significant differences were identified using the Fisher's Least Significant Difference post hoc test. Pearson's correlation coefficient was used to compare the two methods for the measurement of the fNIRS activation magnitude. All data values were reported as mean \pm SD within the text and + one SD for the figures. A statistical significance level of $p<0.05$ was used for all analyses. Effect size was calculated using the Cohen's effect size $d$ (d effects: small $\geq 0.20$, medium $\geq 0.50$, large $\geq 0.80$ ), and is defined as the mean change score divided by the standard deviation of change (Cohen, 1988).

\section{Results}

\subsection{Behavioral data}

The participants' anxiety measured by STAI-YA score increased significantly between the beginning and termination the protocol (29.2 versus 34.2, respectively; $t=4.5 ; p<0.001 ; d=0.6$ ). Performance of the arithmetic task was affected by the difficulty level in terms of the total number of responses $(F r=73.6 ; p<0.001)$, the number of correct answers $(F r=75.5 ; p<0.001)$ and the percent of error rate $(F r=63.6 ; p<0.001)$. Within each condition level, the total number of responses decreased significantly with increasing levels of task difficulty (easy: 38.2 responses, medium: 24.3 responses and difficult: 16.3 responses, respectively). The total number of responses for the difficult level as compared to the easy level was largely reduced by $73 \%(d=3.1)$. We found a mean of $34.6,18.3$ and 9.2 correct answers for the easy, medium and difficult levels, respectively. Error rates were about $10.5 \%, 27.7 \%$ and $45.2 \%$ for the easy, medium and difficult levels, respectively. These first observations were congruent with the mean scores of perceived difficulty $(F=224.4 ; p<0.001)$ where the mean DP15 score was 5.1 (close to "easy"), 8.6 (close to "somewhat difficult"), and 11.5 (close to "difficult") for the easy, medium and difficult levels, respectively. The NASA-TLX scores were gradually higher $(F=59.6 ; p<0.001)$ as a function of the arithmetic task levels ( 43 for easy, 60 for medium, 71 for difficult). Finally, the reaction time (pre-post) significantly changed with the level of difficulty $(F=224.4 ; p<0.001)$. Post-hocs tests indicated a significant increase at the level "difficult" $(p<0.001)$, with corresponding increase as $16.7 \mathrm{~ms}$.

\section{2. fNIRS data and comparative approaches}

Two subjects were excluded because they exhibited quite extreme fNIRS cortical activation during each condition preventing us from doing parametric statistics (i.e., to insure the normality and equal homogeneity of variance in our sample). Hence, in total thirty-six subjects were included in the fNIRS data analyses. In a large sample, when the arithmetic task was difficult, most of our fNIRS responses showed a common cortical activation pattern associated with an increase in oxy-Hb and a slight decrease in deoxy-Hb (Fig. 2). After analyzing 648 fNIRS patterns (36 subjects $\times 2$ hemispheres $\times 3$ conditions $\times 3$ trials), the percent of this type of activation pattern was $63.7 \%$. The remaining pattern was a so-called "deactivation" pattern (i.e., inverse NIRS responses as compared to those observed with typical activation; about $12 \%$ ) or no change (i.e., absence of increase/decrease in any hemoglobin species in either hemisphere between rest and stimulation periods; about 25\%). Comparing fNIRS traces in the right and left PFC, we found no main effect of "hemisphere" by using either the method of changes in amplitude $(F=0.33 ; p=0.57)$ or in slope $(F=0.53$; $p=0.47)$. As a consequence we averaged the two PFCs for all further analyses.

When calculating the slope of the regression line, ANOVA revealed that there was a significant main effect of the level of arithmetic task difficulty ( $F=10.57 ; p<0.001$, see Fig. 3). Post-hoc analyses indicated that slope of oxy-Hb was higher (see Fig. 3 ) for the difficult level compared to the easy level $(p<0.001 ; d=0.65)$ and smaller as compared to the medium level $(p<0.01 ; d=0.32)$. Even if small ( $d=0.31)$, a significant difference was found between the easy and medium levels $(p<0.01)$. No significant difference for deoxy-Hb was observed between the task complexity levels $(p>0.05)$.

Regarding the method of changes in amplitude, there was also a main effect of the difficulty level discriminated by $\operatorname{ANOVA}(F=14.73$; $p<0.001$, see Fig. 3). However, post-hoc tests for oxy-Hb revealed no significant difference between the difficult and medium levels ( $p=0.30 ; d=0.09$ ). But amplitude of oxy-Hb was higher for the difficult level compared to the easy level $(p<0.001 ; d=0.61)$ and smaller for the medium level compared to the easy level $(p<0.001$; $d=0.48)$. No significant difference for deoxy-Hb was observed between all task complexity levels $(p>0.05)$.

Finally, Pearson's correlation coefficients showed strong associations between amplitude vs. slope methods in oxy- $\mathrm{Hb}$ and deoxy-Hb (all $p<0.05$ ). Correlation coefficients for oxy-Hb and deoxy-Hb were respectively, $r=0.85$ and $r=0.9$ for the easy level, $r=0.92$ and $r=0.92$ for the medium level and $r=0.97$ and $r=0.9$ for the difficult level.

\section{Discussion}

\subsection{Experimental results}

The present experimental study was designed to determine the effect of different mental workloads on the behavioral performance and PFC activation using fNIRS. Our fNIRS data indicated that activation in the bilateral PFCs during cognitive tasks corroborates earlier findings using identical arithmetic tasks (Limongi et al., 2009; Sakatani et al., 2010; Tanida et al., 2004, 2007, 2008). The precise conditions that induce prefrontal activation we observed during mental arithmetic task are not well understood but could be associated with working memory, mental stress, or other general attentional and cognitive loads that are not specific to mental arithmetic.

Hock et al. (1995) were the first to adapt the difficulty of the arithmetic task as a function of the calculating abilities for each subject but no explanation was given. In the present study, we 


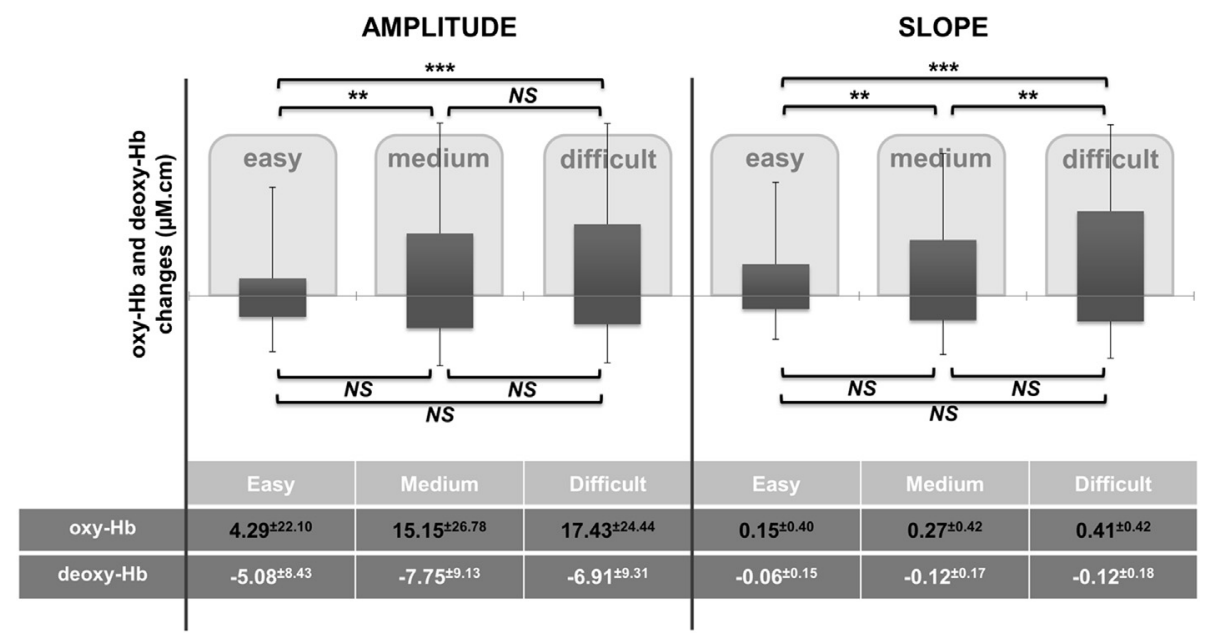

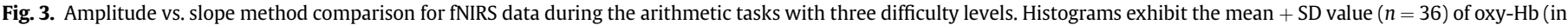

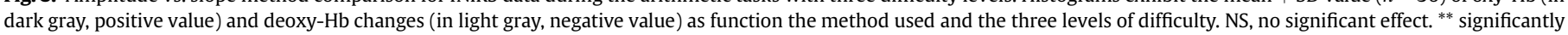
different at $p<.01$ and ${ }^{* * *}$ significantly different at $p<.001$.

insured to evaluate the behavioral performance, the difficulty level, the perceived cognitive load and the PFC activation for each subject. By doing so, we observed a progressive bilateral PFC activation inversely parallel to the fall in cognitive performance. Indeed, an alteration of the performance as a function of the three mental workloads was noted: a dramatic increase in error rate occurred from easy to difficult levels (i.e., from $10.5 \%$ to $45.2 \%$ ). Also, the NASA-TLX and DP15 scores were found to be sensitive to changes in mental workload levels.

Two main other results have to be considered. The first concerns the interesting outcome on RT. The current findings revealed a significant effect on the focused sustained attention for the higher difficult task only. In accordance with the limited capacity theories of attention (Botvinick et al., 2001), such a result suggests that the difficult level of the calculation task required more attentional resources than the two others. It can be suggested that calculating at this level of difficulty consumed too much attentional resources, finally leading up to the draining of the attentional reserve (Wickens, 2008). Further, this experimental result underlines that performing an attentional demanding task can generate a decrement of the performance in other attention-related tasks. Given the substantial role of attention in higher cognitive processes (e.g., working memory, learning; Sarter et al., 2001), such a decrement could crucially impact on activities of daily life. Second, the rise in STAI-YA scores corroborates that somatic adaptation and attentional resources were likely altered during our protocol. Taken together, findings of the present study may be regarded as a major source of fatigue associated with cognitive work. Further studies are required to explore this assumption.

In turn, the level of increase in cortical activation during cognitive task varied with the difficulty of the different levels of calculation for each subject (Fig. 3). The findings of the current study are consistent with those of Tsunashima et al. (2012) who found that the arithmetic task level is reflected by the increase in oxy-Hb. Blood oxygenation increased with increasing workload as long as the subject is attentive and engaged in the task. Our behavioral measures support this assumption. It is expected that during an easy cognitive task there may be less activation of neurons involved. Hence, the hemoglobin oxygenation state changes fewer (Hoshi and Tamura, 1993). In fact, there is a symmetrical effect between the magnitude of the brain activation and the level of cognitive loading where the neural activation is underlying on it
(Hoshi et al., 1994). The present findings seem to be consistent with neuroergonomics research using electrophysiological signals where Gevins and Smith (2008) found EEG changes in response to variations in task difficulty and mental workload during cognitive tasks. During arithmetic tasks, when we compared the results from the task with no difficulty to the more difficult task, the effect size brought to light about a significant large difference between them. Noteworthy that our fNIRS findings are further highlighted using the slope method, which was an alternative approach for discriminating significant differences in cortical activation between the three levels of task difficulty.

\subsection{Comparison between amplitude and slope methods}

This study set out to compare the results between two fNIRS analysis approaches (i.e., amplitude vs. slope methods). Even if the two aforementioned methods of analysis were satisfactory to distinguish different levels of mental workload, we noticed one main difference between them. Statistical results (Fig. 2) showed that the slope coefficient could better discriminate between the medium and difficult conditions whereas it was not possible to do with the amplitude values. Our first expectation for a better discrimination using the slope coefficient was based on the fact that using a linear regression method captures roughly the common profile of the fNIRS data compared to the magnitude of changes of the cortical activation over a pre-defined time window (Gervain et al., 2011). Usually, a baseline period is compared to the largest response obtained during the stimulation period using the amplitude response for oxy- $\mathrm{Hb}$ and deoxy-Hb concentration (Perrey, 2008; Gervain et al., 2011). Since no consensus still exists, the investigators often choose two separated pieces of fNIRS signals with an arbitrary but relevant temporal window (Colier et al., 1999). On the contrary, the slope reflects the linear changes for oxy-Hb and deoxy-Hb during the entire windowed stimulation between the beginning and the final point of the stimulation. By doing so, our findings indicated that the slope for oxy-Hb over the stimulation period was significantly sensitive to task load changes.

Hence, fNIRS analysis by slope seems appropriate to capture some important aspects of the cerebral hemodynamic response during cognitive processes. An exact shape of the hemodynamic response does not exist and various functional forms have already been illustrated. Nonetheless, as suggested by Gervain et al. (2011) 
the dynamic of the typical fNIRS-derived cerebral hemodynamic response is temporally depicted by a small increase in oxy- $\mathrm{Hb}$ concentration, immediately followed by a high increase in oxy$\mathrm{Hb}$, and concomitantly a slight decrease in deoxy-Hb concentration, to achieve a peak or a plateau, before slowly returning to baseline after stimulation. A number of fitting procedures exist that potentially allow for characterization of the latency, magnitude and duration of the fNIRS responses. In the present study, we focus on a simple procedure for extracting an estimation of response height, time-to-peak and full-width as potential measures of response magnitude, latency and duration. Linear regression has the ability to provide independent estimates of multiple parameters of the hemodynamic response with low computational costs. Even if the issue of hemodynamic response modeling is well beyond the scope of this study, our results show that evoked fNIRS-derived cerebral hemodynamic response to cognitive tasks was translated in slope coefficient with enough sensitivity (Figs. 2 and 3). Notably such linear method should pay attention to specific experimental paradigm like long stimulation in block design (i.e., $60 \mathrm{~s}$ in the present study). However, with a short windowed stimulation (e.g., under $10 \mathrm{~s}$ ), caution must be applied, as the slope method might not be transferable. No similar point of view was adopted in the fNIRS literature before. Hence, more future researches on this temporal matter need to be undertaken.

We are aware that the proposed slope method is a very crude mean-field simplification of the fNIRS signals response described earlier. Nevertheless the linear regression represents the first order derivative of the signal exhibiting the typical cortical activation. This can be illustrated by a significant positive value in oxy-Hb concentration and a negative value in deoxy-Hb concentration which are interpreted separately. We believed that the relevance of the slope coefficient sign would enable the fact to use a new indicator for characterizing functional epoch segment during functional motor or cognitive tasks. Similar to the possible calculation of oxygenation index (i.e., oxy-Hb - deoxy-Hb) or blood volume index (i.e., oxy-Hb + deoxy-Hb) initially proposed by Izzetoglu et al. (2004), other indicators based on slope calculation (e.g., slope $\mathrm{oxy}_{\text {- }}$ $\mathrm{Hb}-$ slope $_{\text {deoxy-Hb}}$ ) could be set out. Finally, fNIRS analysis by slope is easy and user-friendly to use because detrending is not necessary.

\section{3. fNIRS limitations}

In the present fNIRS study, we revealed in majority the typical activation pattern observation (around 64\%) characterized by an increase in oxy-Hb concentration and a decrease in deoxy-Hb concentration during the cognitive tasks. Therefore, the remaining percent of fNIRS derived-cerebral responses corresponded to either inverse oxygenation pattern (about $12 \%$ ) or no change. Particularly, in fNIRS literature the term 'deactivation' suggests an antagonist shape in both hemoglobin species (oxy- and deoxy-Hb) (Bauernfeind et al., 2008; Pfurtscheller et al., 2010). This particular cerebral hemodynamic response is likely to reflect individual differences in performance-related signals and may contribute to the commonly observed inter-subject variability in fNIRS measurements (Sato et al., 2005). Even if the interpretation of a deactivation pattern is not clear, some possible explanations for this unexpected oxygenation response could be proposed: (i) extracortical (systemic physiological fluctuations) effects may dominate the fNIRS-derived cortical signals (Bauernfeind et al., 2008; Kirilina et al., 2012); (ii) the location accuracy of the interrogated tissue may change among subjects (the so-called partial volume effects, Boas et al., 2001); (iii) $\mathrm{oxy}-\mathrm{Hb}$ can be drained from the PFC region to be delivered to another near region more activated due to the complexity of neural network implicated in the processing of arithmetic tasks (e.g., parietal areas, Gruber et al., 2001; Power et al., 2010; Pfurtscheller et al.,
2010); (iv) the deactivation patterns could be caused either by "local inhibition" (e.g. inhibitory interneurons) or "attenuation in regional brain activity" (Gusnard and Raichle, 2001; Pfurtscheller et al., 2010).

\section{Conclusion}

On the whole, this investigation endeavored to measure the mental workload at different levels of a cognitive task. We showed that three incremental levels of mental workload could be differentiated suitably with respect to a sensitive fNIRS data analysis. The method by slope we proposed in the present study showed its ability to distinguish sensitively between each levels of task complexity This is a step forward to analyze the hemodynamic response by fNIRS. Future research is warranted to fully explore the advantages and limitations of the linear regression and slope coefficient comparatively to others for quantitative analysis of fNIRS data.

\section{Acknowledgments}

This work was conducted at the Euromov Technological Platform of Montpellier-1 University. It was supported in part by the grant-in-aid for scientific research of the Languedoc-Roussillon Region council, the French University Institute and the National Agency for Research and Technology (No. 1198/2009). The authors are indebted to the participants for their invaluable contribution. We would also like to thank the anonymous reviewers for general comments and insightful interpretation of the results.

\section{References}

Ayaz, H., Shewokis, P.A., Bunce, S., Izzetoglu, K., Willems, B., Onaral, B., 2012. Optical brain monitoring for operator training and mental workload assessment. NeuroImage 59, 36-47.

Bauernfeind, G., Leeb, R., Wriessnegger, S.C., Pfurtscheller, G., 2008. Development, set-up and first results for a one-channel near-infrared spectroscopy system. Biomedical Engineering 53, 36-43.

Boas, D.A., Gaudette, T., Strangman, G., Cheng, X., Marota, J.J., Mandeville, J.B., 2001 The accuracy of near infrared spectroscopy and imaging during focal changes in cerebral hemodynamics. NeuroImage 13 (1), 76-90.

Botvinick, M.M., Braver, T.S., Barch, D.M., Carter, C.S., Cohen, J.D., 2001. Conflict monitoring and cognitive control. Psychological Review 108, 624-652.

Cohen, J., 1988. Statistical Power Analysis for the Behavioral Sciences, second ed. Lawrence Erlbaum, New Jersey.

Colier, W.N.J.M., Quaresima, V., Oeseburg, B., Ferrari, M., 1999. Human motor-cortex oxygenation changes induced by cyclic coupled movements of hand and foot Experimental Brain Research 129, 457-461.

Delignieres, D., Famose, J.P., Genty, J., 1994. Validation of a scale for the assessment of perceived task difficulty. Sciences et Techniques des Activités Physiques et Sportives 34, 77-88.

Di Nocera, F., Camilli, M., Terenzi, M., 2007. A random glance at the flight deck: pilots' scanning strategies and the real-time assessment of mental workload. Journal of Cognitive Engineering and Decision Making 1 (3), 271-285.

Fairclough, S.H., Venables, L., Tattersall, A., 2005. The influence of task demand and learning on the psychophysiological response. International Journal of Psychophysiology 56, 171-184.

Ferrari, M., Quaresima, V., 2012. A brief review on the history of human functional near-infrared spectroscopy (fNIRS) development and fields of application. Neurolmage 63 (2), 921-935.

Gagnon, C., Desjardins-Crépeau, L., Tournier, I., Desjardins, M., Lesage, F., Greenwood, C.E., Bherer, L., 2012. Near-infrared imaging of the effects of glucose ingestion and regulation on prefrontal activation during dual-task execution in healthy fasting older adults. Behavioural Brain Research 232 (1), 137-147.

Gervain, J., Mehler, J., Werker, J.F., Nelson, C.A., Csibra, G., Lloyd-Fox, S., Shukla, M., Aslin, R.N., 2011. Near-infrared spectroscopy: a report from the McDonnell infant methodology consortium. Developmental Cognitive Neuroscience 1, 22-46.

Gevins, A., Smith, M.E., 2008. Electroencephalography (EEG) in neuroergonomics. In: Parasuraman, R., Rizzo, M. (Eds.), Neuroergonomics: The Brain at Work. Oxford University Press, Oxford, pp. 15-31.

Gruber, O., Indefrey, P., Steinmetz, H., Kleinschmidt, A., 2001. Dissociating neural correlates of cognitive components in mental calculation. Cerebral Cortex 11, 350-359.

Gusnard, D.A., Raichle, M.E., 2001. Searching for a baseline: functional imaging and the resting human brain. Nature Reviews Neuroscience 2 (10), 685-694.

Hart, S.G., Staveland, L.E., 1988. Development of NASA-TLX (task load index): results of experimental and theoretical research. In: Hancock, P.A., Meshkati, N. (Eds.), Human Mental Workload. North Holland, Amsterdam, pp. 139-183. 
Heekeren, H.R., Obrig, H., Wenzel, R., Eberle, K., Ruben, J., Villringer, K., Kurth, R. Villringer, A., 1997. Cerebral haemoglobin oxygenation during sustained visua stimulation-a near-infrared spectroscopy study. Philosophical Transactions of the Royal Society B 352, 743-750.

Hirshfield, L., Solovey, E., Girouard, A., Kebinger, J., Jacob, R., Sassaroli, A., Fantini, S., 2009. Brain measurement for usability testing and adaptive interfaces: an example of uncovering syntactic workload with functional near infrared spectroscopy. In: Proceedings of the 27th International Conference on Human Factors in Computing Systems, pp. 2185-2194.

Hock, C., Müller-Spahn, F., Schuh-Hofer, S., Hofmann, M., Dirnagl, U., Villringer, A., 1995. Age dependency of changes in cerebral hemoglobin oxygenation during brain activation: a near-infrared spectroscopy study. Journal of Cerebral Blood Flow and Metabolism 15, 1103-1108.

Holper, L., Biallas, M., Wolf, M., 2009. Task complexity relates to activation of cortical motor areas during uni- and bimanual performance: a functional NIRS study. NeuroImage 46, 1105-1113.

Hoshi, Y., Tamura, M., 1993. Detection of dynamic changes in cerebral oxygenation coupled to neuronal function mental work in man. Neuroscience Letters 150, 5-8.

Hoshi, Y., Onoe, H., Watanabe, Y., Andersson, J., Bergström, M., Lilja, A Langström, B., Tamura, M., 1994. Non-synchronous behavior of neuronal activity, oxidative metabolism and blood supply during mental tasks in man Neuroscience Letters 172, 129-133.

Izzetoglu, K. Bunce, S.C., Onaral, B., Pourrezaei, K., Chance, B., 2004 . Functiona optical brain imaging using near-infrared during cognitive tasks. International Journal of Human-computer Interaction 17, 211-227.

Izzetoglu, M., Bunce, S.C., Izzetoglu, K., Onaral, B., Pourrezaei, K., 2007. Functiona brain imaging using near-infrared technology; assessing cognitive activity in real-life situations. IEEE Engineering in Medicine and Biology Magazine.

Kirilina, E., Jelzow, A., Heine, A., Niessing, M., Wabnitz, H., Brühl, R., Tachtsidis, I., 2012. The physiological origin of task-evoked systemic artefacts in functiona near infrared spectroscopy. NeuroImage 61 (1), 70-81.

León-Carrión, J., León-Domínguez, U., 2012. Functional near-infrared spectroscopy (fNIRS): principles and neuroscientific applications. In: Bright, P. (Ed.), Neuroimaging - Methods. ISBN: 978-953-51-0097-3, InTech, Available from: http:/ www.intechopen.com/books/neuroimaging-methods/functional-near-infraredspectroscopy-fnirs-brain-studies-and-others-clinical-uses.

Limongi, T, Di Sante, G, Ferrari, M., Quaresima, V., 2009. Detecting mental calculation related frontal cortex oxygenation changes for brain computer interface using multi-channel functional near infrared topography. International Society for Bioelectromagnetism 11, 86-90.

Perrey, S., 2008. Non-invasive NIR spectroscopy of human brain function during exercise. Methods 45, 289-299.

Perrey, S., Thedon, T., Rupp, T., 2010. NIRS in ergonomics: Its application in industry for promotion of health and human performance at work. International Journa of Industrial Ergonomics 40, 185-189.
Pfurtscheller, G., Bauernfeind, G., Wriessnegger, S.C., Neuper, C., 2010. Focal frontal (de)oxyhemoglobin responses during simple arithmetic. International Journal of Psychophysiology 76 (3), 186-192.

Pickup, L., Wilson, J., Lowe, E., 2010. The operational demand evaluation checklist (ODEC) of workload for railway signalling. Applied Ergonomics 41, 393-402.

Power, S.D., Falk, T.H., Chau, T., 2010. Classification of prefrontal activity due to mental arithmetic and music imagery using hidden Markov models and frequency domain near-infrared spectroscopy. Journal of Neural Engineering 7, 026002

Ryu, K., Myung, R., 2005. Evaluation of mental workload with a combined measure based on physiological indices during a dual task of tracking and mental arithmetic. International Journal of Industrial Ergonomics 35, 991-1009.

Sakatani, K., Tanida, M., Katsuyama, M., 2010. Effects of aging on activity of the prefrontal cortex and autonomic nervous system during mental stress task. Advances in Experimental Medicine and Biology 662, 473-478.

Sarter, M., Givens, B., Bruno, J.P., 2001. The cognitive neuroscience of sustained attention: where top-down meets bottom-up. Brain Research Reviews 35 146-160.

Sato, H., Fuchino, Y., Kiguchi, M., Katura, T., Maki, A., Yoro, T., Koizumi, H., 2005. Intersubject variability of near-infrared spectroscopy signals during sensorimotor cortex activation. Journal of Biomedical Optics 10 (4), 44001

Spielberger, C.D., Gorsuch, R.L., Lushene, R.E., 1970. State-trait Anxiety Inventory. Consulting Psychologists Press, CA.

Takeuchi, T., 2000. Change in blood volume in the brain during a simulated aircraft landing task. Journal of Occupational Health 42 (2), 60-65.

Tanida, M., Sakatani, K., Takano, R., Tagai, K., 2004. Relation between asymmetry of prefrontal cortex activities and the autonomic nervous system during a mental arithmetic task: near infrared spectroscopy study. Neuroscience Letters 369, 69-74.

Tanida, M., Katsuyama, M., Sakatani, K., 2007. Relation between mental stressinduced prefrontal cortex activity and skin conditions: a near-infrared spectroscopy study. Brain Research 1184, 210-216.

Tanida, M., Katsuyama, M., Sakatani, K., 2008. Effects of fragrance administration on stress-induced prefrontal cortex activity and sebum secretion in the facial skin. Neuroscience Letters 432, 157-161.

Tsunashima, H., Yanagisawa, K., Iwadate, M., 2012. Measurement of brain function using Near-Infrared Spectroscopy (NIRS). In: Bright, P. (Ed.), Neuroimaging Methods. ISBN: 978-953-51-0097-3, InTech, Available from: http://www. intechopen.com/books/neuroimaging-methods/measurement-of-brainfunction-using-near-infrared-spectroscopy-nirs-.

Wickens, C.D. 2008. Multiple resources and mental workload. Human Factors 50, $449-455$.

Yang, H., Wang, Y., Zhou, Z., Gong, H., Luo, Q., Wang, Y., Lu, Z., 2009. Sex differences in prefrontal hemodynamic response to mental arithmetic as assessed by nearinfrared spectroscopy. Gender Medicine 6, 565-574. 